\title{
ARE OUR NURSES HEALTHY? CARDIORESPIRATORY FITNESS IN A VERY EXHAUSTING PROFESSION
}

\author{
Markéta Sovová1, Eliška Sovová1, Marie Nakládalová2, Tereza Pokorná1, Lenka Štégnerová1, Oldřich Masný1, \\ Katarína Moravcová', Ladislav Štěpánek \\ 'Department of Exercise Medicine and Cardiovascular Rehabilitation, Faculty of Medicine and Dentistry, Palacký University Olomouc and \\ University Hospital Olomouc, Olomouc, Czech Republic \\ ${ }^{2}$ Department of Occupational Medicine, Faculty of Medicine and Dentistry, Palacký University Olomouc and University Hospital Olomouc, \\ Olomouc, Czech Republic
}

\begin{abstract}
SUMMARY
Objectives: Low cardiorespiratory fitness (CRF) is related to higher risk of cardiovascular diseases, increase in all-cause mortality and higher risk of different tumors. The reverse is also true; improvement in CRF is related to decrease in mortality. Cardiopulmonary exercise testing (CPET) is a standard and also the most precise test for determination of CRF - the best possibility is the maximal test measuring different parameters including maximal oxygen consumption. Healthcare professionals throughout the developed world have markedly high rates of sickness absence, burnout, and distress compared to other sectors and this leads to higher risk factors. The study aimed to assess CRF in a group of nurses in a big hospital and compare it with population norms and available published results.

Methods: Nurses over 50 years of age working in one faculty hospital were gradually included in the study from the beginning of 2018 . These nurses work in physically demanding positions. A CPET was carried out following the Bruce protocol.

Results: 90 nurses ( 84 females and 6 males), mean age 55.7 years, were evaluated by CPET. The resting blood pressure was within the norm in 58 persons (64.44\%), maximal oxygen consumption in 61 persons $(67.8 \%)$, W/kg in 25 persons $(46.2 \%)$. We detected a hypertension reaction in 28 persons $(31.1 \%)$, some types of arrhythmia in 17 persons $(18.9 \%$ ) and signs of ischaemia in 8 persons $(8.9 \%)$. The result of CPET led to further examination in 42 persons $(46.6 \%)$. Detailed examination resulted in change of medication in 21 nurses $(23.3 \%)$. New diseases were diagnosed in 15 nurses (hypertension, atrial fibrillation, mitral valve prolapse indicated for cardiac surgery, coronary artery stenosis, and lipid disorders).

Conclusions: It was concluded that the usage of CPET during the regular medical check-ups significantly increases detection of hidden diseases and thus improves the care for nurses.
\end{abstract}

Key words: cardiorespiratory fitness, exercise testing, maximal oxygen consumption, nurse

Address for correspondence: L. Štěpánek, Department of Occupational Medicine, University Hospital Olomouc, I. P. Pavlova 185/6, 77900 Olomouc, Czech Republic. E-mail: stepanek.ladislav@gmail.com

https://doi.org/10.21101/cejph.a6210

\section{INTRODUCTION}

Cardiovascular diseases are the leading cause of death in developed countries. During the last three decades, it has been confirmed that low cardiorespiratory fitness (CRF) is related to higher risk of cardiovascular diseases, increase in all-cause mortality and higher risk of different tumors $(1,2)$. On the other hand, improved CRF decreases mortality (3). The American Heart Association published recommendations for assessing CRF in clinical practice: a case for fitness as a clinical vital sign (4) with the conclusion that adding this parameter to the classic risk factors (hypertension, diabetes, lipid disorders, smoking etc.) improves the risk calculation.

Cardiopulmonary exercise testing (CPET) is a standard and also the most precise test for determination of CRF - a test measuring upper limits of different parameters including maximum oxygen consumption $\left(\mathrm{VO}_{2} \max \right)$.

CRF in the general population improved in the 1960s and 1970s but has been progressively declining since the 1980s. All developed countries experienced declines in CRF with a very strong negative correlation between CRF trends and obesity (5). Currently, physical inactivity resulting in both obesity and insufficient CRF is identified as the fourth leading risk factor for global mortality ( $6 \%$ of deaths globally) (6). The connection between physical activity and CRF is self-evident. According to the study of Davidson et al. (7) performed in adult men, physical inactivity is associated with all-cause mortality independent of common risk factors, but not CRF. Conversely, CRF remains a strong predictor of mortality independent of common risk factors including physical activity. Healthcare professionals throughout the developed world have markedly higher rates of sickness absence, burnout, and distress compared to other sectors. There is evidence that the prevalence of overweight and obesity among nurses is increasing with a significant impact on their health and productivity (8). The most important study - Nurses' Health Study (9) analyzed various parameters of nurses' health without $\mathrm{CRF}$ with $\mathrm{VO}_{2}$ max. The aim of this study was to assess CRF in a group of nurses in a big hospital and compare it with population norms and available published results. 


\section{MATERIALS AND METHODS}

Nurses over 50 years of age working in one faculty hospital were gradually included in the study from the beginning of 2018 according to the following criteria.

Inclusion criteria: working in a physically demanding profession, signing an informed consent form. Exclusion criteria: acute respiratory illness, high rest blood pressure (BP), not achieving the maximal effort - respiratory exchange ratio (RER) more than $1.05, \mathrm{VO}_{2}$ max curve in plateau, termination of test for other reasons than personal maximum.

All subjects underwent clinical examination including casual blood pressure, pulse rate, oxygen saturation, weight and height measurements, BMI (weight divided by height squared) calculations, and ECG.

Maximal testing using the Bruce protocol was performed (BTL equipment - bicycle ergometry, breath by breath measurement) with measurement of the following parameters in rest and maximal effort: BP (2-minute interval, manual measurement), continual monitoring of heart rate, oxygen consumption, carbon dioxide production, RER, ratio $\mathrm{VO}_{2} / \mathrm{VCO}_{2}$, load (Watt and $\mathrm{W} / \mathrm{kg}$ ). Maximal oxygen consumption and load in $\mathrm{W} / \mathrm{kg}$ were compared with population norms $(10,11)$ for age and sex.

The hypertensive reaction was defined as an increase in systolic BP more than $10 \mathrm{~mm} \mathrm{Hg} /$ metabolic equivalent (MET) (12). The relevant guidelines were used for the definition of hypertension (13).

Because of a small number of men in the group, we did not investigate the correlation between gender and results. If there was a pathological finding on CPET (BP, ECG) an additional examination was done.

Table 1. Basic characteristics of the study sample $(N=90)$

\begin{tabular}{|c|c|}
\hline & Mean (SD) \\
\hline Weight (kg) & $77.3(15.6)$ \\
\hline Height (cm) & $166.7(6.7)$ \\
\hline BMI & $27.8(5.4)$ \\
\hline BP rest systolic $(\mathrm{mmHg})$ & $136.5(18.3)$ \\
\hline BP rest diastolic $(\mathrm{mmHg})$ & $84.1(9.4)$ \\
\hline HR rest & $81.8(11.8)$ \\
\hline $\mathrm{HR} \max$ & $153.6(17.6)$ \\
\hline BP max systolic (mmHg) & $187.4(23.4)$ \\
\hline BP max diastolic (mmHg) & $89.2(15.1)$ \\
\hline $\mathrm{VO}_{2} \max$ (peak) & $1691(417)$ \\
\hline $\mathrm{VO}_{2} \max$ (peak) $/ \mathrm{kg} / \mathrm{min} / \mathrm{mL}$ & $21.9(5.4)$ \\
\hline $\mathrm{VCO}_{2} \max$ (peak) & $1927(657)$ \\
\hline Respiratory exchange ratio & $1.2(0.14)$ \\
\hline $\mathrm{VE} / \mathrm{NCO}_{2}$ & $29.7(4.5)$ \\
\hline Metabolic equivalent & $6.9(2.1)$ \\
\hline W & $144.3(46.7)$ \\
\hline W/kg & $1.91(0.55)$ \\
\hline
\end{tabular}

$\mathrm{HR}$ - heart frequency; $\mathrm{VO}_{2}$ max - maximum oxygen consumption;

$\mathrm{VCO}_{2} \mathrm{max}$ - maximal carbon dioxide production; $\mathrm{VE}$ - maximal ventilation

\section{RESULTS}

Ninety nurses ( 84 females and 6 males), mean age 55.7 years, were evaluated by CPET; 28 nurses $(31.1 \%)$ had BMI in a normal range, 36 nurses $(40.0 \%)$ were overweight, 26 nurses $(28.9 \%)$ were obese, and 3 nurses had BMI more than 40 (3.33\%). The range of BMI in the sample was 19.55-45.0.

Forty-three nurses $(47.8 \%)$ suffered from arterial hypertension on medication, $3(3.3 \%)$ from type 2 diabetes mellitus on medication, 1 nurse $(1.1 \%)$ from stable coronary artery disease, 13 nurses (14.4\%) from lipid disorders on medication, 15 nurses $(16.7 \%)$ from thyroid disorders on medication, 3 nurses $(3.3 \%)$ from paroxysmal atrial fibrillation, 3 nurses $(3.3 \%)$ had a history of malignant tumor, 3 nurses $(3.3 \%)$ were treated for asthma, and 1 nurse $(1.1 \%)$ had a history of pulmonary embolism. Only 8 nurses $(8.9 \%)$ had no history of any disease. The description of the studied parameters is shown in Table 1.

Maximal oxygen consumption was within a normal range in 59 persons $(65.6 \%)$, under the norm in $29(32.2 \%)$, and above the norm in 2 persons $(2.2 \%)$. MET value was in 30 persons $(33.3 \%)$ under 6 (tolerance of heavy work). Value of $\mathrm{W} / \mathrm{kg}$ was within the norm in 25 persons $(46.2 \%)$. There were no population norms available for 36 nurses (40\%). The resting BP was within the norm in 58 persons $(64.4 \%)$. During the test, we detected a hypertension reaction in 28 persons ( $31.1 \%$ ), of them 18 persons $(20.0 \%)$ had a history of hypertension. ECG findings - some types of arrhythmia (premature supraventricular or ventricular beats) were noted in 17 persons $(18.9 \%)$ and ischaemia (horizontal ST depression $\geq 2 \mathrm{~mm}$ ) in 8 persons $(8.9 \%)$. The result of CPET led to further examination of 42 persons (46.6\%) (Table 2). More detailed examination resulted in a change of medication in 21 nurses $(23.3 \%)$.

New diseases were diagnosed in 15 nurses: 4 nurses $(4.4 \%)$ arterial hypertension, 2 nurses $(2.2 \%)$ masked hypertension, 4 nurses (4.4\%) new onset of atrial fibrillation, 1 nurse (1.1\%) severe mitral valve prolapse indicated for cardiac surgery, 1 nurse $(1.1 \%)$ coronary artery stenosis, and 3 nurses $(3.3 \%)$ lipid disorder. One nurse took anticoagulation with the control of INR one year ago, 6 nurses did not participate in the recommended evaluation.

\section{DISCUSSION}

Our work revealed two important findings. Firstly, CRF nearly in one third of nurses was under the population norm

Table 2. Overview of subsequent examinations

\begin{tabular}{|l|c|}
\hline & Number \\
\hline Ambulatory blood pressure monitoring & 26 \\
\hline 24 hour ECG monitoring & 19 \\
\hline Calcium score & 6 \\
\hline Single-photon emission computed tomography & 2 \\
\hline CT coronarography & 7 \\
\hline Echocardiography & 29 \\
\hline Coronarography & 2 \\
\hline
\end{tabular}


which took age and sex into account and their performance met only requirements of middle work (3-6 MET). It is questionable whether these persons can continue to work in such a demanding profession. Secondly, the results of CPET in our nurses necessitated further examinations of some nurses that led to new diagnoses.

To the first finding, $\mathrm{CRF}$ with $\mathrm{VO}_{2}$ consumption measurement is a very important prognostic parameter (3). CRF protects against stress-related symptoms, burnout and depression (14). In our group, the poor CRF in one third of nurses can predict worse cardiovascular prognosis. The results can be partly influenced by the weight because only one third of the nurses had BMI under 25 (the normal weight), but the overweight and obesity is another risk factor for cardiovascular morbidity and mortality.

We used Czech population norms - reference values for the study because there are not any globally used norms. In a recent systemic review of 29 studies (15) (87,256 subjects) on reference values for CPET parameters (14 studies using bicycle ergometry, 14 using a treadmill, 1 both), no single set of ideal reference values was found. Authors concluded that the harmonization of CPET data is still urgently needed and recommend FRIEND as an excellent source for CPET reference values (16).

When comparing our results with the literature, the largest study which evaluated the state of health of nurses is the Nurses Health Study (9). This study confirmed decreasing mortality in persons with moderate physical activity which obviously relates to CFR, but they did not measure the CRF. Similarly, Fair et al. (17) compared the occurrence of risk factors in nurses working in prevention care with the results of the abovementioned study and they confirmed fewer risk factors in the preventive care nurse group. The Baptist Health South Florida study (18) is one of the biggest studies of the health status in healthcare professionals, but this study did not use the $\mathrm{VO}_{2}$ max parameter to measure CRF. They used the American Heart Association (AHA) score system. The study included 34,746 employees between 2011 and 2014. The ideal cardiovascular health was determined in only $0.3 \%$ of employees in 2011 and $0.6 \%$ of employees in 2014.

Apropos CRF examination in other professions, Khazraee et al. (19) studied a group of 115 fire-fighting personnel, but the average age in this group was 32 years and thus we could not compare the results with our group. The same was true for Lyytikäinen et al. (20) who studied rescuers (average age 34, $\mathrm{VO}_{2} \max 51 \mathrm{~mL} / \mathrm{kg} / \mathrm{min}$ ). Kettunen et al. (21) did a submaximal exercise testing with an estimation of $\mathrm{VO}_{2} \max$ in a group of 371 workers. The average age was 45 years and the average estimated value of $\mathrm{VO}_{2} \max$ was $32.1 \mathrm{~mL} / \mathrm{kg} / \mathrm{min}$. Considering the age differences, we were unable to use the figures for comparison with our group. Jirák et al. (22) observed CRF in mining rescuers. One third did not meet the population $\mathrm{VO}_{2} \max$ norms which confirms our results but there were only male persons participating in this study.

Genin et al. studied CRF in a group of sedentary office workers (23) without measurement of $\mathrm{VO}_{2} \max$. The resting heart rate in the group of 193 office employees (mean age 44.2 years) in the active group was 67 beats/min and in the inactive group 71 beats/min. In our group, the resting heart rate was
81.8 beats/min. This difference could be explained by chronic stress in our group.

The second finding is, that the results of CPET in our nurses necessitated further examinations of some nurses that led to new diagnoses. We found no comparable research and we assume that the reason is that if a problem is determined during CPET, further examination is carried out in various other departments whose statistical data are not available. Further medical problems in our nurses may be caused by a high level of stress (24), shift work (25), a higher number of smokers compared to the general public (26), and sickness (27).

Many nurses display unhealthy eating and physical activity behaviours resulting in an increasing rate of overweight. As an example, half of nurses in Canada do not meet current recommendations for physical activity. Obesity in nurses has been associated with a wide range of negative outcomes, such as productivity loss, occupational injuries, and musculoskeletal disorders $(8,28)$. In our study, the average BMI was in the overweight range and the higher rest heart rate does not suggest that it is a sample of individuals engaged in regular physical activity. However, a physical activity questionnaire was not a part of the study.

In our sample, several observed persons did not return for a medical check, one nurse even used anticoagulation for one year without any control. People who were diagnosed with new diseases mostly reported that they have had problems for a long time.

What is the relationship of health professionals to their health? We do not have much data on this problem. Sickness presenteeism is a behaviour in which an employee is physically present at work with reduced performance due to illness or other reasons. Hospital doctors and nurses are more inclined to exhibit presenteeism than other professional groups, resulting in diminished staff health, reduced team productivity and potentially higher indirect presenteeism-related medical costs than absenteeism (27).

In our work, we compared the incidence of risk factors in hospital staff and insurance company staff some time ago (29). Comparison of these two groups of professionals showed that all the insurance company employees came to have the medical check contrast to hospital staff.

How to help improve the situation? One possibility is "physical activity programme", but the literature describes success (21) as well as a very low adherence, e.g. Genin et al. (23) report adherence in $45 \%$ of office workers. The influence of lifestyle modification on cardiometabolic risk factors was described by Das et al. in a group of healthcare employees with 2 type diabetes (30). The intervention improved the values of weight, BP, lipid profile and HbA. Kelly et al. (8) in their review focused on primary studies concerning lifestyle interventions to address obesity in nurses. All 11 available studies reported positive changes in participants' health behaviours, albeit small in some cases. A significant reduction of BMI was noted only in 4 studies and a significant positive change in physical activity appeared in 5 studies.

A limitation of the study may be an insufficient focus on the sample characteristics in terms of evaluating the personal level of free-time physical activity and also of activity intensity at the workplace. 


\section{CONCLUSIONS}

CRF nearly in one third of the study sample was under the population norm. It was concluded that the usage of CPET during the regular medical check-ups of staff significantly increases the detection of hidden diseases and thus improves the care for nurses.

\section{Acknowledgements}

This work was supported by the Ministry of Health, Czech Republic conceptual development of research organization (Palacký University Olomouc, 61989592, University Hospital Olomouc, 00098892).

\section{Conflict of Interests}

None declared

\section{REFERENCES}

1. Schmid D, Leitzmann MF. Cardiorespiratory fitness as predictor of cancer mortality: a systematic review and meta-analysis. Ann Oncol. 2015;26(2):272-8.

2. Kodama S, Saito K, Tanaka S, Maki M, Yachi Y, Asumi M, et al. Cardiorespiratory fitness as a quantitative predictor of all-cause mortality and cardiovascular events in healthy men and women: a meta analysis. JAMA. 2009 May 20;301(19):2024-35.

3. Lee DC, Artero EG, Sui X, Blair SN. Mortality trends in the general population: the importance of cardiorespiratory fitness. J Psychopharmacol. 2010 Nov;24(4 Suppl):27-35.

4. Ross R, Blair SN, Arena R, Church TS, Després JP, Franklin BA, et al. Importance of assessing cardiorespiratory fitness in clinical practice: a case for fitness as a clinical vital sign: a scientific statement from the American Heart Association. Circulation. 2016;134(24):e653-99.

5. Lamoureux NR, Fitzgerald JS, Norton KI, Sabato T, Tremblay MS, Tomkinson GR. Temporal trends in the cardiorespiratory fitness of 2,525,827 adults between 1967 and 2016: a systematic review. Sports Med. 2019;49(1):41-55.

6. World Health Organization. Global strategy on diet, physical activity and health [Internet]. Geneva: WHO; 2019 [cited 2020 Feb 27]. Available from: https://www.who.int/dietphysicalactivity/pa/en/.

7. Davidson T, Vainshelboim B, Kokkinos P, Myers J, Ross R. Cardiorespiratory fitness versus physical activity as predictors of all-cause mortality in men. Am Heart J. 2018;196:156-62.

8. Kelly M, Wills J. Systematic review: What works to address obesity in nurses? Occup Med (Lond). 2018;68(4):228-38.

9. Yu E, Rimm E, Qi L, Rexrode K, Albert CM, Sun Q, et al. Diet, lifestyle, biomarkers, genetic factors, and risk of cardiovascular disease in the Nurses' Health Studies. Am J Public Health. 2016;106(9):1616-23.

10. Seliger V, Bartůněk $Z$. Mean values of various indices of physical fitness in the investigation of Czechoslovak population aged 12-55 years. Prague: Czechoslovak Association of Physical Culture; 1976.

11. Mácek M, Seliger V, Vávra J, Skranc O, Horák J, Piric M, et al. Physical fitness of the Czechoslovak population between the ages of 12 and 55 years. Oxygen consumption and pulse oxygen. Physiol Bohemoslov. 1979;28(1):75-87.

12. Fletcher GF, Ades PA, Kligfield P, Arena R, Balady GJ, Bittner VA, et al. Exercise standards for testing and training: a scientific statement from the American Heart Association. Circulation. 2013;128(8):873-934.

13. Williams B, Mancia G, Spiering W, Rosei EA, Azizi M, Burnier M, et al. $2018 \mathrm{ESC} / \mathrm{ESH}$ guidelines for the management of arterial hypertension. Kardiol Pol. 2019;77(2):71-159. (In Polish.)
14. Gerber M, Lindwall M, Lindegård A, Börjesson M, Jonsdottir IH. Cardiorespiratory fitness protects against stress-related symptoms of burnout and depression. Patient Educ Couns. 2013;93(1):146-52.

15. Takken T, Mylius CF, Paap D, Broeders W, Hulzebos HJ, Van Brussel M, et al. Reference values for cardiopulmonary exercise testing in healthy subjects - an updated systematic review. Expert Rev Cardiovasc Ther. 2019;17(6):413-26.

16. Kaminsky LA, Imboden MT, Arena R, Myers J. Reference standards for cardiorespiratory fitness measured with cardiopulmonary exercise testing using cycle ergometry: data from the fitness registry and the importance of exercise national database (FRIEND) registry. Mayo Clin Proc. 2017;92(2):228-33.

17. Fair JM, Gulanick M, Braun LT. Cardiovascular risk factors and lifestyle habits among preventive cardiovascular nurses. J Cardiovasc Nurs. 2009;24(4):277-86.

18. Ogunmoroti O, Utuama O, Spatz ES, Rouseff M, Parris D, Das S, et al. Trends in ideal cardiovascular health metrics among employees of a large healthcare organization (from the Baptist Health South Florida Employee Study). Am J Cardiol. 2016;117(5):787-93.

19. Khazraee T, Fararouei M, Daneshmandi H, Mobasheri F, Zamanian Z. Maximal oxygen consumption, respiratory volume and some related factors in fire-fighting personnel. Int J Prev Med. 2017 Apr 13;8:25. doi: 10.4103/ijpvm.IJPVM_299_16.

20. Lyytikäinen K, Toivonen L, Hynynen E, Lindholm H, Kyröläinen H. Recovery of rescuers from a 24 -h shift and its association with aerobic fitness. Int J Occup Med Environ Health. 2017;30(3):433-44.

21. Kettunen O, Vuorimaa T, Vasankari T. A 12-month exercise intervention decreased stress symptoms and increased mental resources among working adults - Results perceived after a 12-month follow-up. Int J Occup Med Environ Health. 2015;28(1):157-68.

22. Jirák Z, Lvončík S, Tomášková H, Buzga M, Trlicová M, Tekletová D. Criteria for evaluating working physical fitness of mine rescuers. Prac Lek. 2013;65(1-2):6-13. (In Czech.)

23. Genin PM, Dessenne P, Finaud J, Pereira B, Dutheil F, Thivel D, et al. Effect of work-related sedentary time on overall health profile in active vs. inactive office workers. Front Public Health. 2018 Oct 1;6:279. doi: 10.3389/fpubh.2018.00279.

24. Duffy E, Avalos G, Dowling M. Secondary traumatic stress among emergency nurses: a cross-sectional study. Int Emerg Nurs. 2015;23(2):53-8.

25. Ferri P, Guadi M, Marcheselli L, Balduzzi S, Magnani D, Di Lorenzo $\mathrm{R}$. The impact of shift work on the psychological and physical health of nurses in a general hospital: a comparison between rotating night shifts and day shifts. Risk Manag Healthc Policy. 2016;9:203-11.

26. Kaletová M, Sovová E, Nakládalová M, Benušová I, Doupalová P, Lukl J. Smoking in nurses. Prac Lek. 2005;57(2):87-90. (In Czech.)

27. Lui JNM, Andres EB, Johnston JM. Presenteeism exposures and outcomes amongst hospital doctors and nurses: a systematic review. BMC Health Serv Res. 2018 Dec 19;18(1):985. doi: 10.1186/s12913-018-3789-z.

28. Power BT, Kiezebrink K, Allan JL, Campbell MK. Understanding perceived determinants of nurses' eating and physical activity behaviour: a theory-informed qualitative interview study. BMC Obes. 2017 May 9;4:18. doi: 10.1186/s40608-017-0154-4.

29. Sovová E, Nakládalová M, Kaletová M, Lukl J, Benušová I, Doupalová P. Determination of occurrence of risk factors of cardiovascular diseases in the stafff of an insurance company as a part of preventive examination: Does this group differ from health professionals? Prac Lek. 2006;58(1): 5-8. (In Czech.)

30. Das S, Rouseff M, Guzman HE, Osondu CU, Brown D, Betancourt $\mathrm{B}$, et al. Impact of lifestyle modification on cardiometabolic risk factors in health-care employees with type 2 diabetes. Am J Health Promot. 2019;33(5):745-8.

Received March 25, 2020

Accepted in revised form August 2, 2020 